INVITED PAPER

\title{
Generation of dark solitons by interaction between similaritons in Raman fiber amplifiers
}

\author{
Christophe FINOT $^{1}$, John M. DUDLEY ${ }^{2}$ and Guy MILLOT ${ }^{1}$ \\ Christophe.Finot@u-bourgogne.fr \\ ${ }^{1}$ Laboratoire de Physique de l'Université de Bourgogne, Centre National de la Recherche \\ Scientifique, Unité Mixte de Recherche 5027, 21078 Dijon, France \\ ${ }^{2}$ Département d'Optique P. M. Duffieux, Institut FEMTO-ST, Unité Mixte de Recherche 6174, \\ Université de Franche-Comté, 25030 Besançon, France
}

\begin{abstract}
We present a new method to generate dark soliton trains by exploiting the interaction between two time-delayed optical similaritons with the same wavelength. The temporal overlap of two similariton pulses creates a sinusoidal beating which subsequently evolves into an ultrahigh repetition-rate train of dark solitons through the combined effects of normal dispersion, non-linearity and adiabatic Raman gain. The experimental results are in good agreement with numerical predictions. We also investigate how the the repetition rate of the dark soliton train depends on the time separation between the initial pulses, the initial pulse energy, and the Raman gain. Finally, we numerically study the interaction between three similaritons of the same wavelength.
\end{abstract}

Running head: Interaction between similaritons

Author to whom proofs should be sent: Guy MILLOT, LPUB - UMR CNRS 5027

9 Av. A. Savary, BP 47870, 21078 Dijon Cedex, France.

Tel.: 33 (0)3 803959 81; Fax: 33 (0)3 8039 59 71; E-mail : Guy.Millot@u-bourgogne.fr 


\section{Introduction}

The application of self-similarity techniques to the study of nonlinear pulse propagation has been the subject of much recent interest in the context of parabolic pulse generation in optical fiber amplifiers with normal group-velocity dispersion (GVD) [1-18]. Such pulses, also called optical similaritons, represent a new class of solutions to the non-linear Schrödinger equation (NLSE) with gain. They are asymptotically generated in the amplifier, independently of the shape or noise of the input pulse $[3,12]$, and propagate self-similarly subject to exponential scaling of their pulse amplitude, temporal duration and spectral width [5]. In this case, the interplay of normal dispersion, nonlinearity and gain produces a linearly chirped pulse with a parabolic intensity profile [3].

Similaritons are of wide-ranging practical significance since their intrinsic resistance to the deleterious effects of the wave breaking [1] allows the scaling of amplifiers to higher-power regimes $[19,20]$. Moreover, their linear chirp facilitates efficient temporal compression $[3,18$ 20]. Combination of a similariton amplifier with an optical feedback has resulted in a new regime of laser mode-locking that is likely to have major implications for the development of high-power ultrashort pulse laser oscillators [21]. Recent experimental studies have also taken advantage of the similariton characteristics to propose new methods for optical pulse synthesis [22], for $10 \mathrm{GHz}$ telecom multiwavelength sources [23] or for optical regeneration of telecom signal [24]. The experimental works have mainly relied on rare-earth doped fiber amplifiers with dopants such as Erbium [2,23] or Ytterbium [3,19-21]. However, it has been shown that a resonant amplification is not a necessary requirement for parabolic pulse generation and that Raman scattering could be efficiently used as amplification process $[8,9,13]$. This opens up the possibility to provide gain at wavelengths where no conventional doped amplifiers are possible. 
To date, most of the theoretical and experimental studies have been devoted to the dynamics of single similariton pulses. The dynamics of the amplification of a pair of pulses is more complex. In particular, during the amplification process, the exponential self-similar scaling of the temporal duration of each individual pulse leads to a temporal overlap and interaction of the pulses after sufficient propagation distance. As similariton-based techniques have been recently demonstrated in the field of telecommunication $[23,24]$ it has become crucial to better understand the various phenomena which can be involved in the case of a similariton overlap during the propagation [25].

We present in this paper a detailed study of the interactions occurring in the overlap region of a pair of optical similaritons of the same wavelength generated in a Raman amplifier where the amplification is nearly-adiabatic. Our amplifier at $1550 \mathrm{~nm}$ relies on a normally dispersive Non-Zero Dispersion-Shifted Fiber (NZ-DSF) in association with a commercial wattlevel CW pump source $[8,13]$. We first detail the numerical predictions obtained using a model based on the Non-Linear Schrödinger Equation (NLSE) with a constant gain term. In particular, we demonstrate that the pulses evolve independently to generate self-similar parabolic profiles, only interacting in their overlap region. At the beginning of their overlap the interaction between the two linearly chirped parabolic pulses is almost linear and leads to a frequency beating of the intensity profile. After the first stage of overlap, however, the beating, subject to the combined effects of non-linearity, normal dispersion and adiabatic Raman gain, evolves into a train of dark solitons. We show that dark solitons also appear when taking into account the longitudinal and frequency dependences of the gain, as well as higher order linear and non-linear terms in the NLSE. We then compare quantitatively the results of the computations with experimental data. We also carry out a detailed study of the dependence of the repetition rate of dark-soliton train 
on the characteristics of the input pulse pair and the gain of the amplifier. Finally we briefly consider the interaction between three similaritons of the same wavelength.

\section{Numerical simulations}

\subsection{Basic model}

We first present results from simulations that demonstrate numerically the generation of a train of dark solitons under typical experimental conditions. Evolution of the slowly varying complex envelope $\psi$ of an initial pulse during its propagation in a normal-dispersion Raman amplifier can be modeled by the NLSE with a constant longitudinal and spectral gain $g$ :

$$
i \frac{\partial \psi}{\partial z}=i \frac{g}{2} \psi+\frac{\beta_{2}}{2} \frac{\partial^{2} \psi}{\partial t^{2}}-\gamma|\psi|^{2} \psi
$$

where $\beta_{2}>0$ and $\gamma$ are the second-order dispersion and Kerr nonlinear coefficients, respectively. The asymptotic similariton solution of Eq.(1) in the limit $z \rightarrow \infty$ is characterized by a parabolic intensity profile and a positive linear chirp, whose analytical expression is given by [3]:

$$
\begin{gathered}
\psi_{p}(t, z)=A_{P}(z) \sqrt{1-\left(\frac{t}{T_{p}(z)}\right)^{2}} \exp \left[i\left(\varphi_{p}(z)-\pi C_{P} t^{2}\right)\right] \quad,|t| \leq T_{p} \\
\psi_{p}(t)=0 \quad,|t| \geq T_{p}
\end{gathered}
$$

Here $\varphi_{p}$ is a phase offset. The solution (2) corresponds to a compactly-supported pulse whose zero-crossing points are given by $T_{p}(z)=\left(6 A_{0} / g\right)\left(\gamma \beta_{2} / 2\right)^{1 / 2} \exp (g z / 3)$, with $A_{o}$ a constant amplitude term $A_{o}=1 / 2\left[g U_{\text {ini }} /\left(\gamma \beta_{2} / 2\right)^{1 / 2}\right]^{1 / 3}$, where $U_{\text {ini }}$ is the initial pulse energy. These parabolic pulses have a peak-power given by $\left|A_{p}\right|^{2}=A_{0}{ }^{2} \exp (2 g z / 3)$ and a positive linear chirp with the slope $C_{p}=g /\left(6 \pi \beta_{2}\right)$. We note that the temporal width $T_{p}$ of the pulse exponentially increases with the propagation length $z$. 


\subsection{Similariton propagation outside the overlap region}

We now consider the amplification of a pair of identical pulses separated by a time-delay $\Delta T=$ 55.5 ps. The time origin of the copropagating frame is the temporal centre of gravity of the pair of initial pulses, so that the pulses are centered at $t= \pm \Delta T / 2$. Each initial pulse has a chirp-free Gaussian intensity profile with a duration of $5 \mathrm{ps}$, a carrier wavelength of $1550 \mathrm{~nm}$ and an initial energy of $2.9 \mathrm{pJ}$. The Raman amplifier parameters at $1550 \mathrm{~nm}$ are $\gamma=2.0 \times 10^{-3} \mathrm{~W}^{-1} \mathrm{~m}^{-1}$ and $\beta_{2}$ $=4.6 \times 10^{-3} \mathrm{ps}^{2} \mathrm{~m}^{-1}$. The linear gain coefficient $\mathrm{g}$ is $0.972 \times 10^{-3} \mathrm{~m}^{-1}$, which leads, for an amplifier length of $5.3 \mathrm{~km}$, to a total integrated gain of $22.3 \mathrm{~dB}$. Figure 1(a) shows the evolution of the pair of pulses as a function of the propagation distance in the amplifier, as calculated from numerical integration of Eq. (1). The solid lines show the total evolution of the incident pair of pulses and, for comparison, the open circles show the expected evolution of one pulse propagating on its own. We note that there is no noticeable difference between the intensity profile of a single pulse (circles) and the intensity profile of a pulse in the presence of its neighbor (solid line). Therefore, both before and after the pulses overlap, each pulse evolves separately with a parabolic profile, and only interact in the overlap region. Owing to their compact nature, parabolic pulses are clearly unaffected by their neighbor outside the overlap region. In consequence, their mean temporal positions remain constant along the amplifier. This is in contrast to the case of two interacting solitons, where both the position and shape of the pulses are modified by the interaction.

\subsection{Modulation of the intensity profile in the overlap region}

Figure 1 (a) clearly shows that, at a distance of $4.17 \mathrm{~km}$, an oscillation appears inside the overlap. Indeed, as the parabolic pulses are linearly chirped, the frequency difference between 
the overlapping leading and trailing edges of the two pulses induces a beating in the resultant signal. This is shown more clearly in Fig. 1(b) (solid line).

To understand the characteristics of this beating in more detail, we first neglect the nonlinear effects which occur in the interaction process between the two similaritons. If we consider that the pulses have developed a parabolic shape as described by Eq. (2), the overlap region extends from $t=\Delta T / 2-T_{p}$ to $t=T_{p}-\Delta T / 2$. The resulting intensity profile in the overlap region will be thus given by the linear superposition of the falling edge of the first pulse, whose instantaneous frequency is $f_{0}+C_{p}(t+\Delta T / 2)$, with the raising edge of the second pulse whose instantaneous frequency is $f_{0}+C_{p}(t-\Delta T / 2)$ :

$$
\psi_{\text {pair }}(t)=A_{P}\left\{\begin{array}{l}
\sqrt{1-\left(\frac{t+\Delta T / 2}{T_{p}}\right)^{2}} \exp \left[-i \pi C_{P}(t+\Delta T / 2)^{2}\right] \\
+\sqrt{1-\left(\frac{t-\Delta T / 2}{T_{p}}\right)^{2}} \exp \left[-i \pi C_{P}(t-\Delta T / 2)^{2}\right]
\end{array}\right\},
$$

where the phase offset terms were neglected. We will thus see a sinusoidal beating due to the superposition of the two instantaneous frequencies separated by $f_{s}=C_{p} \Delta T$. The intensity in the overlap region is then given by:

$$
\left|\psi_{\text {pair }}(t)\right|^{2}=2\left|A_{P}\right|^{2}\left\{\begin{array}{l}
1-\frac{1}{T_{p}^{2}}\left(t^{2}+\frac{\Delta T^{2}}{4}\right) \\
+\cos \left(2 \pi C_{P} t \Delta T\right) \sqrt{1-\left(\frac{t+\Delta T / 2}{T_{p}}\right)^{2}} \sqrt{1-\left(\frac{t-\Delta T / 2}{T_{p}}\right)^{2}}
\end{array} .\right.
$$

We thus have $N=f_{s}\left(2 T_{p}-\Delta T\right)$ oscillations which appear in the overlap region. At the center of the overlap region (when $|t| \ll T_{p}-\Delta T / 2$ ), the intensities of the pulses are comparable so that near $100 \%$ modulation is expected. At the inner edges of the overlap region, 
the contributions of the pulses become different, so that the modulation depth decreases. The circles in Fig. 1(b) represent the linear superposition of the similaritons (Eq. 4) after $4.17 \mathrm{~km}$ of propagation, resulting in oscillation with a modulation frequency $f_{s}=C_{p} \Delta T=622 \mathrm{GHz}$. Figure 1(b) shows that, at the center of the overlap region, this oscillation is in good agreement with the numerical simulations of Eq. (1) (solid line). However, it is significant that the oscillations predicted by numerical simulations extend over a larger range than those obtained from simple linear superposition of the two parabolic pulses : the value of $N$ obtained by numerical simulations is then superior to $f_{s}\left(2 T_{p}-\Delta T\right)$. These additional oscillations, which originate in the wings of the similariton pulses [5,6], implicitly appear from numerical integrations of Eq. (1), but are neglected by assuming that the pulses have an exact parabolic shape (Eq. (4)). In our case, the large apparent value of the wings is related to the fact that, after $4.17 \mathrm{~km}$ of propagation, the pulses are not completely in the asymptotic regime.

\subsection{Generation of high-repetition rate trains of dark solitons}

When the overlapping pulses further propagate through the amplifier subject to the combined effects of non-linearity, normal dispersion and adiabatic Raman gain, the oscillation reshapes into a train of dark solitons [26]. Such a reshaping effect has been previously exploited in the spatial field with the transformation of a periodic sinusoidal modulation into a spatial array of dark solitons [27]. The normalized intensity profile of a dark soliton is given by [28] $I(T)=$

$A^{2}\left(1-B^{2} \operatorname{sech}^{2} T\right) / B^{2}$, where $A^{2}$ is the intensity depth of the hole, $B^{2}$ is a blackness factor related to the contrast and $T$ is a dimensionless retarded time. Dark solitons with $B^{2}=1$ have been called black, whereas other dark solitons with $-1<B<1$ are called gray. The blackness parameter $|B|$ can be calculated from the values of the power and phase jump at the dip center. For example, 
for the dark soliton designed as (1) in Fig. 1(c), the blackness parameter is $|B|=0.997 \cong 1$ and the phase jump is $0.97 \pi \cong \pi$, so that this hole is very close to a black soliton with a $\tanh ^{2}$ shape. Indeed, Fig. 1(c) confirms that after $5.3 \mathrm{~km}$ of propagation, the intensity and phase profiles of the dip (1) (solid line) are in very good agreement with the corresponding profiles of a black soliton (circles) with a temporal width $T_{d}=385 \mathrm{fs}$, a pulse depth $P_{d}=15.6 \mathrm{~W}$ and a phase jump of $\pi$. Taking into account that the fundamental black soliton depth is $P_{o}=\beta_{2} /\left(\gamma T_{d}^{2}\right)=15.9 \mathrm{~W} \cong P_{d}$, we may conclude that the characteristics of dip (1) are very close to those of a fundamental black soliton.

The repetition rate of the dark-soliton train after a propagation distance of $5.3 \mathrm{~km}$ is $f_{d}=380$ GHz. Figure 2(a) shows the spectrum of the dark soliton train (solid line) after $5.3 \mathrm{~km}$ of propagation, compared with that of a single pulse (dashed grey line). As can be seen from Fig. 2(a), the generation of a train of dark solitons is characterized by the apparition of peaks, with a frequency spacing equal to $f_{d}$. The growth of these spectral peaks can be seen as a manifestation of a four-wave mixing process.

Figure 2(b) represents the evolution of the contour plot of the normalized intensity profile of the dark soliton train with further amplification distance. The time-shifts of the dips during propagation, which are clearly observed if Fig. 2(b), indicate that dark solitons propagate at different relative velocities $\beta$ with respect to the group velocity at the initial wavelength. It has been previously shown that $\beta$ has the same sign as $B$ and is proportional to $A \sqrt{1-B^{2}} / B$ [28]. On the other hand $B>0(B<0)$ when the phase increases (decreases) across the pulse [28]. So, according to the phase profile shown in Fig. 1(c), at $t<0(B<0)$ the dark soliton velocity 
decreases, whereas at $t>0(B>0)$ the dark soliton velocity increases, in agreement with the numerical results of Fig. 2(b). Since $|\beta|$ is a decreasing function of $|B|$, the highest relative velocities $|\beta|$ are expected at the edges of the overlap region. Moreover, since $|\beta|$ is an increasing function of $A$, the relative velocities $|\beta|$ increase with the amplification distance. So, as can be seen in Fig. 2(b), the time separation between the dark soliton centers do not grow larger in a linear fashion with the propagation distance along the amplifier. Indeed, the repetition rate of the soliton train rapidly decreases from 380 to $135 \mathrm{GHz}$ when the propagation distance only increases from 5.3 to $7.3 \mathrm{~km}$. It is noteworthy to remark from Fig. 2(b) that the temporal width of the dark solitons decreases with propagation distance, e.g. from $380 \mathrm{fs}$ at $5.3 \mathrm{~km}$ to 200 fs at $7.3 \mathrm{~km}$. As a matter of fact, the solitons adiabatically adapt their temporal width in order to satisfy the relationship between the peak power and the duration during the amplification process [29]. There is a global conservation of the area of the dark soliton pulse. This temporal compression is different from the spreading of the dark solitons, as described by Rothenberg [30] in the case of the generation of a train of dark solitons by the colliding of two pulses. In the scheme of Ref. [30], the physical phenomenon responsible for the overlap of the pulses was the temporal broadening induced in a passive fiber by the deleterious effect of optical wavebreaking.

\section{Use of a more realistic model}

The model (Eq. (1)) used in the previous section was based on the assumption of a constant longitudinal gain and neglects several effects which can affect the shape of the output pulse, such as the longitudinal dependence of the gain profile $[4,17]$ or the dispersion of the Raman gain [31,32]. For a more accurate modelization of our experimental results, we present now 
simulations using the generalized extended NLSE [31] that rigorously includes the Raman amplification process through an appropriate integral term, as well as effects such as higherorder dispersion terms and self-steepening :

$$
\begin{aligned}
& i \frac{\partial \psi}{\partial z}=-i \frac{\alpha}{2} \psi+\frac{\beta_{2}}{2} \frac{\partial^{2} \psi}{\partial t^{2}}+i \frac{\beta_{3}}{6} \frac{\partial^{3} \psi}{\partial t^{3}} \\
& -\gamma\left[1+\frac{i}{\omega_{s}} \frac{\partial}{\partial t}\right] \psi(z, t) \int_{0}^{\infty} R\left(t^{\prime}\right)\left|\psi\left(z, t-t^{\prime}\right)\right|^{2} d t^{\prime}
\end{aligned} .
$$

The function $R(t)=\left(1-f_{r}\right) \delta(t)+f_{r} h_{r}(t)$ includes the instantaneous electronic response and the delayed Raman contribution $h_{r}(t)$, with the fractional Raman contribution $f_{r}=0.18$. For $h_{r}(t)$, we used the measured Raman response of fused silica [32]. The third order dispersion is $\beta_{3}=1.0 \mathrm{x}$ $10^{-6} \mathrm{ps}^{3} \mathrm{~m}^{-1}$. The wavelength dependent loss coefficient $\alpha$ is also included in Eq. (5). Here, $\psi(z, t)$ $=\psi_{s}(z, t)+\psi_{r}(z, t) \exp (-i \Omega t)$, where $\psi_{s}$ and $\psi_{r}$ are the slowly varying envelopes of signal and pump fields oscillating at $\omega_{s}$ and $\omega_{r}$, respectively. $\Omega=\omega_{r}-\omega_{s}$ is the pump-signal frequency detuning chosen so that near optimal Raman gain for the signal is achieved at $1550 \mathrm{~nm}$. The initial conditions $\psi_{s}(0, t)$ for the signal are given by the intensity and chirp of the two input pulses directly measured by using a frequency-resolved optical gating (FROG) technique [33]. The pump $\psi_{r}$ is assumed to be a noise free continuous wave at $1455 \mathrm{~nm}$. We use a counterpropagating amplification scheme, which has the advantage to be only slightly affected by the time-dependent pump depletion effects [17]. In consequence, in that pump configuration, the two input signal pulses have nearly the same Raman gain. On the contrary, pump depletion effects, which would occur in a forward pumping geometry, would lead to different effective Raman gain for the two signal pulses. The pump power is adjusted in order to provide a total integrated gain of $22.3 \mathrm{~dB}$ for the signal. This corresponds to a power $\left|\psi_{r}(L)\right|^{2}$ of $0.95 \mathrm{~W}$. 
In practice, to improve the numerical efficiency (and thus reduce the computation time), assuming a continuous Raman pump, we used the following set of two coupled extended NLSE's [13,17] with the subscripts $s$ and $r$ indicating the signal and Raman pump wavelengths respectively:

$$
\begin{gathered}
i \frac{\partial \psi_{s}}{\partial z}=-i \frac{\alpha_{s}}{2} \psi_{s}+\frac{\beta_{2}}{2} \frac{\partial^{2} \psi_{s}}{\partial t^{2}}+i \frac{\beta_{3}}{6} \frac{\partial^{3} \psi_{s}}{\partial t^{3}} \\
-\gamma\left[1+\frac{i}{\omega_{s}} \frac{\partial}{\partial t}\right]\left\{\left(1-f_{r}\right) \psi_{s}\left(\left|\psi_{s}\right|^{2}+2\left|\psi_{r}\right|^{2}\right)\right. \\
+f_{r} \psi_{s} \int_{0}^{\infty} h\left(t^{\prime}\right)\left(\left|\psi_{s}\left(z, t-t^{\prime}\right)\right|^{2}+\left|\psi_{r}\left(z, t-t^{\prime}\right)\right|^{2}\right) d t^{\prime} \\
\left.+f_{r} \psi_{r} \int_{0}^{\infty} h\left(t^{\prime}\right) \psi_{s}\left(z, t-t^{\prime}\right) \psi_{r}^{*}\left(z, t-t^{\prime}\right) e^{-i \Omega t} d t^{\prime}\right\} \\
\frac{\partial \psi_{r}}{\partial z}=\frac{\alpha_{r}+\alpha_{d}(z)}{2} \psi_{r}
\end{gathered}
$$

The influence of pump depletion on the longitudinal pump power evolution is taken into account by the introduction of a term $\alpha_{d}(z)$, which can be numerically determined by an iterative process based on an average power analysis [34]. The generalized split-step Fourier method was used to numerically solve Eq. (6) [31].

Figures 3(a) and 3(b) show the phase and intensity profiles (solid lines) obtained from numerical integration of Eq. (6) at a propagation distance of $5.3 \mathrm{~km}$. The intensity profile of the initial pulses retrieved from FROG measurements is displayed in Fig. 3(a) by the dashed line. In Fig. 3(b), the deepest hole is in good agreement with a least-squares fit of a $\tanh ^{2}$ function (circles) with a temporal width of $T_{d}=0.42 \mathrm{ps}$. We also observe in Fig. 3(b) a brutal phase jump of a value close to $\pi$ at the minimum of the pulse, which is the signature of a dark (black) soliton. The spectrum of the pair of pulses displayed in Fig. 3(c) is similar to that resulting from the 
model with a constant gain (Fig. 2(a)). The frequency separation between two consecutive peaks indicates a repetition rate of $446 \mathrm{GHz}$ instead of $380 \mathrm{GHz}$ obtained from Eq. (2). So we can conclude that the simple model with a constant gain (Eq. (1)) gives a correct qualitative prediction of the consequences of the interaction between the two similaritons. However, an accurate quantitative comparison between numerical calculations and experimental results requires the use of the extended NLSE (Eq. (6)).

\section{Experimental results}

\subsection{Experimental setup}

Figure 4 shows a schematic representation of the experimental setup. The $1550 \mathrm{~nm}$ input pulse was obtained from a Pritel passively mode-locked fiber laser operating at a repetition rate of 22 MHz. The initial pulse had a nearly Fourier transform-limited intensity profile with a temporal duration of 5 ps (see section 4.2). The pulses emitted from the picosecond laser were sent through a beamsplitter and recombined in a Mach-Zehnder interferometer with a variable delay between the two pulses. A variable attenuator placed in one arm of the interferometer allows us to obtain two pulses with the same energy, whereas a polarizer located at the interferometer output permits to obtain two pulses with the same linear state of polarization. The Raman amplifier consists in $5.3 \mathrm{~km}$ of NZ-DSF, whose parameters have been reported in section 2.2. The fiber was pumped in a backward configuration [17] by a continuous wave Raman laser (Keopsys), delivering a power of $1420 \mathrm{~mW}$ at $1455 \mathrm{~nm}$. A WDM coupler with high power rating permitted the superposition of pump and signal beams. An optical isolator at $1480 \mathrm{~nm}$ was inserted after the polarizer to prevent that part of the residual pump in the Raman amplifier 
disturbs the operation of the picosecond laser. Input and output pulses were characterized by their optical spectrum and autocorrelation. When possible (according to the pulse energy and recording time), the pulse intensity and phase were characterized using a FROG device based on second harmonic generation [13]. The phase and intensity retrieval was performed using the generalized projections algorithm [33]. The reliability of the FROG measurements was checked using the standard techniques based on comparisons of the independently-measured autocorrelation and spectrum with the FROG trace marginals [33].

\subsection{Characterization of a single similariton}

We have first characterized using FROG the initial pulses whose retrieved intensity profile is shown in Fig. 5 by diamonds. Figure 5 (circles) also shows the retrieved intensity and chirp profiles of the pulse at the amplifier output in the case of a single initial pulse. As can be seen in Fig. 5, the experimental intensity and chirp profiles are in very good agreement with parabolic and linear fits, respectively (gray dashed line). The output pulse exhibits the expected characteristics of a similariton with a total temporal width $2 T_{p}=73.5 \mathrm{ps}$, a chirp slope $C_{p}=$ $0.0118 \mathrm{THz} / \mathrm{ps}$ and a peak power of $10 \mathrm{~W}$. The experimental data are also compared with numerical integration of Eq. (6) (solid line). As can be observed in Fig. 5, there is a very good agreement between numerical and experimental results, confirming the reliability of the model described by Eq. (6). It is noteworthy that the model based on the assumption of a constant gain (Eq. (1)) leads to significantly different parameters: $2 T_{p}=85 \mathrm{ps}$ and $C_{p}=0.0107 \mathrm{THz} / \mathrm{ps}$. The assumption of a constant gain is clearly not the most appropriate to accurately reproduce the characteristics of the similariton as observed experimentally. As a result, we have only used the numerical integrations of Eq. (6) in what follows. 


\subsection{Characterization of the interaction between two similaritons}

We now characterize the similariton interaction in the case of an initial pair of pulses with an energy of $2.9 \mathrm{pJ}$ and a temporal separation of $\Delta T=55.5 \mathrm{ps}$. We note that, as the dark solitons generated during the similariton interaction are expected to have a temporal width of only $T_{0}=$ $0.32 \mathrm{ps}$, pulse characterization using FROG would require both a large total temporal window (about 200 ps) as well as a high temporal resolution (about $20 \mathrm{fs}$ ) to completely define the pulse structure. This would necessitate measurement of a very large number of data points and a corresponding long acquisition time. Unfortunately, owing to the lack of long term stability of our FROG device, we were not able to process a direct FROG characterization of light at the amplifier output. It would also be very difficult to implement a cross-correlation technique similar to that used in Refs. $[30,35,36]$ since our initial pulses are much more broader than the dark solitons we would like to characterize. However, it was possible to characterize the generation of the dark soliton train via interaction of two parabolic pulses by direct measurements of the autocorrelation and spectrum of light at the amplifier output.

Figure 6(a) shows the autocorrelation trace recorded at the center of the overlap region (circles). The experimental trace, which exhibits $410-\mathrm{GHz}$ oscillations, is in good agreement with numerical integrations of the extended NLSE (Eq. (6)). The experimental data are also compared with a sinusoidal fit (dotted line). We can observe from Fig. 6(a) a large disagreement between the experimental results and the sinusoidal fit, which is a clear signature of the nonlinear reshaping of the sinusoidal modulation from the onset of the similariton interaction into a train of dark solitons. The experimental output spectrum, displayed in Fig. 6(b), exhibits several sidebands separated by $407 \mathrm{GHz}$, consistent with the repetition rate measured on the 
autocorrelation signal. We note that that the small spectral asymmetry, which is also present in the experimental output spectrum of a single similariton (dashed gray line), is mainly due to the asymmetry of the initial pulse spectrum (not shown here), slightly accentuated by the effects of Raman intra-pulse scattering. The experimental spectrum is also in good agreement with the corresponding numerical spectrum, shown in Fig. 3(c), obtained from integration of the extended NLSE (Eq. (6)). In view of the excellent agreement between numerical and experimental spectrum and autocorrelation, we can conclude that our results provide clear experimental evidence for the signature of the generation of a train of dark solitons arising from the interaction between two optical similaritons.

\section{Control of the repetition rate of the dark soliton train}

In this section, we investigate both numerically and experimentally the influence of several parameters on the repetition rate $f_{d}$ of the dark soliton train generated from the interaction between two similaritons. We will show that the repetition rate can be controlled by specific choices of the initial time separation $\Delta T$, pulse energy $U_{i n i}$ or integrated gain $G$ of the amplifier. The repetition rate has been determined by measuring the spectral separation of the characteristic sidebands of the spectrum of the two similaritons. Figure 7 shows the evolution of the repetition rate of the train of dark solitons as a function of $\Delta T(a), U_{i n i}(\mathrm{~b})$ and $G(\mathrm{c})$. The experimental results (circles) are compared with numerical integrations of the extended NLSE (Eq. (6)) (solid line) and of the NLSE with a constant gain (Eq. (1)) (dashed line), and with the frequency $f_{s}=C_{p}$ $\Delta T$ of the precursory sinusoidal oscillation resulting from linear overlap of the similariton pulses (mixed line). 


\subsection{Influence of the initial time separation $\Delta T$}

In this study the initial pulse energy and integrated gain were fixed to $2.9 \mathrm{pJ}$ and $22.3 \mathrm{~dB}$, respectively. Figure 7(a) shows that the repetition rate $f_{d}$ is a quasi-linear function of $\Delta T$. The experimental results (circles) are both in good agreement with numerical simulations based on Eq. (6) (solid line) and Eq. (1) (dotted line). As expected, the frequency $f_{d}$ is lower than $f_{s}$ due to the transverse velocity $|\beta|$ of the dark solitons. The difference $\delta=f_{s}-f_{d}$ decreases with $\Delta T:$ for $\Delta T=47 \mathrm{ps}, \delta=270 \mathrm{GHz}$, whereas for $\Delta T=70 \mathrm{ps}, \delta$ is only $180 \mathrm{GHz}$. Since the propagation distance at which the pulses begin to overlap increases with $\Delta T$, dark solitons are generated after a longer distance in the amplifier as $\Delta T$ increases. In consequence, after their generation dark solitons will propagate with their own velocities $|\beta|$ on a shorter distance, so that $\delta$ will be lower (see Fig. 2(b)). The results of Fig. 7(a) clearly demonstrate that the repetition rate $f_{d}$ of the dark soliton train can be precisely adjusted just by changing the time separation $\Delta T$ between the initial pulses.

\subsection{Influence of the initial pulse energy $U_{\text {ini }}$}

With the time separation between the initial pulses being now fixed to $55.5 \mathrm{ps}$, we now change the value the initial pulse energy $U_{\text {ini }}$. The experimental values (circles) shown in Fig. 7(b) show that $f_{d}$ only slightly decreases with $U_{i n i}$. The experimental results are in good agreement with the numerical simulations of both models (solid and dotted lines). The global behavior of $f_{d}$ versus $U_{\text {ini }}$ can be explained by similar considerations that those used in section 5.1 . We recall that the frequency $f_{s}=C_{p} \Delta T$ of the oscillation created at the beginning of the similariton overlap does not depend on the pulse energy. Indeed the chirp parameter $C_{p}$ is independent of $U_{i n i}$ in the 
asymptotic regime. On the other hand, the temporal width $T_{p}$ of the similariton increases with the initial energy (see section 2.1), so that the distance at which the similaritons overlap decreases with $U_{i n i}$. In consequence, after their generation dark solitons will propagate with their own velocities $|\beta|$ on a longer distance, so that the frequency of the periodic train of dark solitons will decrease (see Fig. 2(b)). Another contribution to the decrease of $f_{d}$ with $U_{i n i}$ comes from the fact that the depth of the dark soliton increases with $U_{i n i}$ (see section 2.4) and the velocity $|\beta|$ increases with the soliton depth.

\subsection{Influence of the integrated gain $G$}

We now study the variation of the repetition rate of the dark soliton train as a function of the integrated gain of the Raman amplifier. The initial pulse energy and time delay between the input pulses were fixed to $2.9 \mathrm{pJ}$ and $55.5 \mathrm{ps}$, respectively. We experimentally change the pump power so that the integrated gain $G$ varies from 50 to 170 (17 to $22.3 \mathrm{~dB})$. We can see from Fig. 7(c) that the experimental values of $f_{d}$ (circles) remain nearly constant. Those results are consistent with the evolution predicted by numerical simulations based on the Eq. (6). On the other hand the NLSE with a constant gain (Eq. (1)) predicts calculated values of $f_{d}$ which are slightly underestimated as the integrated gain increases. This can be explained by the fact that the effects of pump depletion become more significant as the integrated gain increases.

The very small dependence of $f_{d}$ versus $G$ is rather unexpected since the frequency of the initial beating $f_{s}$ increases with the linear gain $\mathrm{g}$ in the fiber (mixed line): $f_{s}=C_{p} \Delta T$ with $C_{p}$ directly proportional to $g$ (see section 2.1 ). So, the global evolution of $f_{d}$ versus $G$ can not be only explained by the dependence of $f_{s}$. The velocity $|\beta|$ of dark solitons and the distance over which dark solitons will propagate after their generation play a significant role in the dependence of $f_{d}$ 
versus $G$. Indeed, higher the gain is, lower is the propagation distance at which the overlap of the similaritons occurs and higher is $\delta=f_{s}-f_{d}$. Moreover, the increase of the depth of the dark soliton with $G$ induces an increase of the velocity $|\beta|$ and consequently an increase of $\delta$. Thus the evolution of $f_{d}$ versus $G$ results in a balance between the increases of $f_{s}$ and $\delta$.

\section{Interaction between three similaritons}

Finally, we investigate using numerical simulations the case of the interaction between three similaritons of the same wavelength and temporally separated by same quantity $\Delta T_{o}=35$ ps. The characteristics of the three initial pulses and Raman amplifier are identical to those considered in section 2.2. Figure 8(a) shows that the three initial pulses are transformed into similaritons, characterized by a parabolic intensity profile, before overlapping. Two distinct overlap regions clearly appear at a propagation distance of about $3.3 \mathrm{~km}$. Similarly to the case of the interaction between two similaritons, oscillations appear within each overlap region and further reshape into dark solitons for longer propagation distances. Figure 8(b) shows that at a distance of about $5 \mathrm{~km}$ both overlap regions overlap themselves and collisions of dark solitons appear. It is remarkable to observe from Fig. 8(b) that dark solitons are stable against collisions. To illustrate this property, Fig. 8(c) shows an example of a collision between two dark solitons that occurs at a propagation distance of $5992 \mathrm{~m}$ (solid line) within the time window spreading from -6 ps to -2 ps. We clearly observe from Fig. 8(c) that the intensity profiles of the two colliding dark solitons before the collision (dashed line) remain unchanged after the collision (dotted line). On the other hand, Fig. 8(d) shows that a temporal shift of the dark solitons appears during the collision [28]. 


\section{Conclusions}

In conclusion, we have analyzed the dynamics of the self-similar amplification of a pair of timedelayed identical pulses of the same wavelength in a Raman amplifier. During the amplification process, due to the exponential scaling of their temporal duration, each pulse broadens, so that, after a given distance of propagation, the two pulses overlap and interact. Owing to their compact nature, similaritons are clearly unaffected by their neighbor outside the overlap region. Inside the overlap region, the superposition of the linearly-chirped pulses creates an oscillation. Due to the combination of non-linearity and gain, this oscillation adiabatically evolves into a finite-extent high-repetition rate train of dark solitons. Our experimental results are in good agreement with theoretical calculations and confirm the generation of dark-soliton train at high repetition rates. We have also investigated the evolutions of the repetition rate of the dark solitons as a function of the initial pulse temporal separation, pulse energy and integrated gain. These evolutions are both affected by the intrinsic properties of the similaritons and the relative velocities of the dark solitons. Finally, we have shown that stable collisions of dark solitons appear during the interaction of three similaritons.

Although our experimental results have been obtained using Raman amplification, our conclusions can be extended to other amplification processes as long as we restrict ourselves to normal dispersion, and we are the regime of an adiabatic amplification regime. For example, dark solitons could also be generated via similariton interaction in ultra-long erbium-doped fibers with controlled dispersion properties [37]. 


\section{Acknowledgments}

The authors would like to thank Stéphane Pitois and Cyril Billet for fruitful discussions. This work has been supported by the Fond National pour la Science (FNS) under contract "ACIPhotonique PH43", the Centre National de la Recherche Scientifique (CNRS), and the Conseil Régional de Bourgogne. 


\section{Biographies}

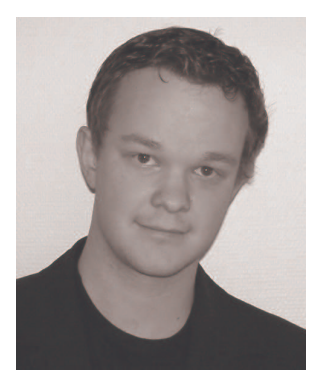

Christophe Finot was born in Chatillon sur Seine, France, in 1978. He received the DEA degree and the engineer degree from the Ecole Superieure d'Optique, Orsay, France in 2002. In 2002, he began a Ph D. at the University of Burgundy, Dijon on optical parabolic pulses. He has published over 20 contributions in journals and conference proceedings.

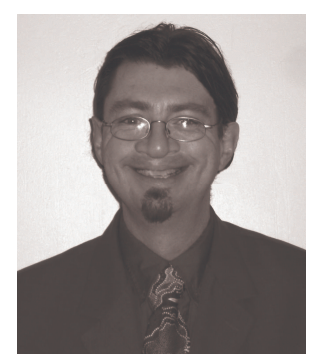

John M. Dudley John Dudley was born in Otahuhu, New Zealand in 1966. He received the B.Sc and Ph.D. degrees in physics from the University of Auckland, New Zealand, in 1987 and 1992 respectively. In 1992 and 1993, he carried out postdoctoral research studying femtosecond selfmodelocked lasers and optical parametric oscillators at the University of St Andrews, Scotland. In 1994, he took up a lecturing position at the University of Auckland where he studied the physics of ultrashort pulse generation and measurement, and nonlinear fiber optics. In 2000, he was appointed a Professor at the University of Franche-Comté in Besançon, France where he is currently carrying out research in ultrafast optics, high speed optoelectronics and optical 
communications. He has published over 170 contributions in journals, books and conference proceedings. Prof. Dudley is a Member of the IEEE and the Optical Society of America. He is a member of the Institut Universitaire de France (IUF).

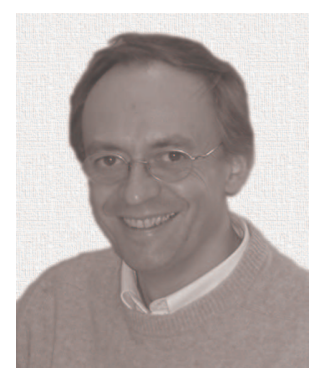

Guy Millot was born in Alligny-en-Morvan, France, in 1960. He received the Ph.D. degree on laser Raman spectroscopy in gases, from the University of Burgundy, Dijon, in 1986. In 1987, he carried out post-doctoral research on time-resolved infrared double resonance experiments in methane and silane at the Massachusetts Institute of Technology. Since 1988, he has been an Assistant Professor and since 1994, a Full Professor in the Physics Department at the University of Burgundy. From 1988 to 1995, he carried out research on nonlinear spectroscopy, molecular structures, time-resolved Raman-infrared double resonance experiments, collisional effects on Raman line-shapes for temperature and concentration measurements in combustion media. Since 1995, his research interests are focused on nonlinear effects in optical fibers, modulational instabilities, solitons, generation, propagation and characterization of optical pulse trains at high repetition rates, stimulated Raman scattering, frequency conversion and applications in optical communications. He has published over 180 contributions in journals, books and conference proceedings. G. Millot was a member of the Institut Universitaire de France (IUF) in the period 2000-2005. He obtained the Silver Medal of the CNRS in 2005. 


\section{References}

1. D. Anderson, M. Desaix, M. Karlson, M. Lisak, and M. L. Quiroga-Teixeiro, "Wavebreaking-free pulses in nonlinear optical fibers", J. Opt. Soc. Amer. B 10 (1993), 11851190.

2. K. Tamura and M. Nakazawa, "Pulse compression by nonlinear pulse evolution with reduced optical wave breaking in erbium-doped fiber amplifiers," Opt. Lett. 21 (1996), $68-70$.

3. M. E. Fermann, V. I. Kruglov, B. C. Thomsen, J. M. Dudley, and J. D. Harvey, "SelfSimilar Propagation and Amplification of Parabolic Pulses in Optical Fibers", Phys. Rev. Lett. 84 (2000), 6010-6013.

4. V.I. Kruglov, A.C. Peacock, J.M. Dudley, and J.D. Harvey, "Self-similar propagation of high-power parabolic pulses in optical fiber amplifiers," Opt. Lett. 25 (2000), 1753-1755.

5. V. I. Kruglov, A. C. Peacock, J. D. Harvey, and J. M. Dudley, "Self Similar Propagation of parabolic pulses in normal-dispersion fiber amplifiers," J. Opt. Soc. Amer. B 19 (2002), 461469.

6. S. Boscolo, S.K. Turitsyn, V.Y. Novokshenov, and J.H.B. Nijhof, "Self-similar parabolic optical solitary waves," Theor. Math. Phys. 133 (2002), 1647-1656.

7. A.C. Peacock, R.J. Kruhlak, J.D. Harvey, and J.M. Dudley, "Solitary pulse propagation in high gain optical fiber amplifiers with normal group velocity dispersion," Opt. Commun. 206 (2002), 171-177.

8. C. Finot, G. Millot, C. Billet, and J.M. Dudley, "Experimental generation of parabolic pulses via Raman amplification in optical fiber," Opt. Express 11 (2003), 1547-1552. 
9. A.C. Peacock, N.G.R. Broderick, and T.M. Monro, "Numerical study of parabolic pulse generation in microstructured fibre Raman amplifiers," Opt. Commun. 218 (2003), 167172.

10. V.I. Kruglov, D. Méchin, and J.D. Harvey, "Self-similar solutions of the generalized Schrödinger equation with distributed coefficients," Opt. Express 12 (2004), 6198-6207.

11. V.I. Kruglov, A.C. Peacock, and J.D. Harvey, "Exact self-similar solutions of the generalized nonlinear Schrödinger equation with distributed coefficients," Phys. Rev. Lett. 90 (2004), 113902.

12. C. Finot, G. Millot, and J. M. Dudley, "Asymptotic characteristics of parabolic similariton pulses in optical fiber amplifiers," Opt. Lett. 29 (2004), 2533-2535.

13. C. Finot, S. Pitois, G. Millot, C. Billet, and J.M. Dudley, "Numerical and experimental study of parabolic pulses generated via Raman amplification in standard optical fibers," IEEE J. Select. Top. Quantum Electron. 10 (2004), 1211-1218.

14. G. Chang, A. Galvanauska, H.G. Winful, and T.B. Norris, "Dependence of parabolic pulse amplification on stimulated Raman scattering and gain bandwith," Opt. Lett. 29 (2004), 2647-2649.

15. T. Hirooka and M. Nakazawa, "Parabolic pulse generation by use of a dispersiondecreasing fiber with normal group-velocity dispersion," Opt. Lett. 29 (2004), 498-500.

16. S. Chen and L. Yi, "Chirped self-similar solutions of a generalized nonlinear Schrödinger equation model," Phys. Rev. E 71 (2005), 016606.

17. C. Finot, "Influence of the pumping configuration on the generation of optical similaritons in optical fibers," Opt. Comm. 249 (2005), 553-561. 
18. C. Billet, J.M. Dudley, N. Joly, and J.C. Knight, "Intermediate asymptotic evolution and photonic bandgap fiber compression of optical similaritons around $1550 \mathrm{~nm}$," Opt. Express 13 (2005), 3236-3241.

19. J. P. Limpert, T. Schreiber, T. Clausnitzer, K. Zöllner, H. J. Fuchs, E. B. Kley, H. Zellmer, and A. Tünnermann, "High-power femtosecond Yb-doped fiber amplifier", Opt. Express 10 (2002), 628-638.

20. A. Malinowski, A. Piper, J. H. V. Price, K. Furusawa, Y. Jeong, J. Nilsson, and D. J. Richardson, "Ultrashort-pulse Yb3+ fiber based laser and amplifier system producing > 25 W average power", Opt. Lett. 29 (2004), 2073-2075.

21. F. Ö. Ilday, J. R. Buckley, W. G. Clark, and F. W. Wise, "Self-similar evolution of parabolic pulses in a laser", Phys. Rev. Lett. 92 (2004), 213902.

22. C. Finot and G. Millot, "Synthesis of optical pulses by use of similaritons", Opt. Express 12 (2004), 5104-5109.

23. Y. Ozeki, Y. Takushima, K. Aiso, K. Taira, and K. Kikuchi, "Generation of $10 \mathrm{GHz}$ similariton pulse trains from $1,2 \mathrm{~km}$-long erbium-doped fibre amplifier for application to multi-wavelength pulse sources", Electron. Lett. 40 (2004), 1103-1104.

24. C. Finot, S. Pitois, and G. Millot, "Regenerative 40-Gb/s wavelength converter based on similariton generation", Opt. Lett. 30 (2005) 1776-1778.

25. C. Finot and G. Millot, "Interaction between optical parabolic pulses in a Raman fiber amplifier," Opt. Express 13 (2005), 5825-5830.

26. E. M. Dianov, P. V. Mamyshev, A. M. Prokorov, and S. V. Chernikov, "Generation of a train of fundamental solitons at a high repetition rate in optical fibers", Opt. Lett. 14 (1989), 1008-1010. 
27. P. V. Mamyshev, C. Bosshard, and G. I. Stegeman, "Generation of a periodic array of dark spatial solitons in the regime of effective amplification", J. Opt. Soc. Amer. B 11 (1994), 1254-1260.

28. R. N. Thurston and A. M. Weiner, "Collisions of dark solitons in optical fibers", J. Opt. Soc. Amer. B 8 (1991), 471-477.

29. W. Zhao and E. Bourkoff, "Generation of dark solitons under a cw background using waveguide electro-optic modulators", Opt. Lett. 15 (1990), 405-407.

30. J. E. Rothenberg, "Dark soliton trains formed by visible pulse collisions in optical fibers", Opt. Commun. 82 (1991), 107-111.

31. G. P. Agrawal, Nonlinear Fiber Optics, Third Edition (San Fransisco, CA : Academic Press, 2001).

32. R. H. Stolen, J. P. Gordon, W. J. Tomlinson, and H. A. Hauss, "Raman response function of silica-core fibers", J. Opt. Soc. Amer. B 6 (1989), 1159-1166.

33. R. Trebino, Frequency-Resolved Optical Gating : The Measurement of ultrashort laser pulses (Norwell, MA : Kluwer Academic Publishers, 2000).

34. B. Min, W. J. Lee, and N. Park, "Efficient formulation of Raman amplifier propagation equations with average power analysis", IEEE Photon. Technol. Lett. 12 (2000), 14861488.

35. P. Emplit, J.P. Hamaide, F. Reynaud, C. Froehly, A. Barthelemy, "Picosecond steps and dark pulses through nonlinear single mode fibers", Optics Comm. 62 (1987), 374-379.

36. A.M Weiner, J.P. Heritage, R.J. Hawkings, R.N. Thurston, E.M. Kirschner, D.E. Leaird and W.J. Tomlinson "Experimental observation of the fundamental dark soliton in optical fibers", Phys. Rev. Lett 61 (1988), 2445-2448. 
37. M. Nakazawa, Y. Kimura, and K. Suzuki, "Ultralong dispersion-shifted erbium-doped fiber amplifier and its application to soliton transmission", IEEE J. Quantum Electron. 26 (1990), 2103-2108. 


\section{Figure captions}

Fig. 1: (a) Numerical calculations of the intensity profiles of a single pulse (circles) and a pair of pulses (solid lines) at different distances of propagation. (b) Central part of the pulse overlap at $z=4.17 \mathrm{~km}$ : simulations (solid line), linear superposition (circles). (c) Intensity and phase profiles at $z=5.3 \mathrm{~km}$ : numerical simulations (solid lines) and black soliton fit (circles).

Fig. 2: (a) Calculated spectra of a single pulse (dashed gray line) and of the pair of pulses (solid line) after $5300 \mathrm{~m}$ of propagation. (b) Contour plot of the evolution of the normalized intensity profile of the dark soliton train from 5.3 to $7.3 \mathrm{~km}$.

Fig. 3: Numerical simulations based on the extended NLSE of the propagation of the pair of pulses after $5300 \mathrm{~m}$ of propagation. (a) Intensity profiles at the amplifier output (solid line). The experimental intensity profile of the initial pulses retrieved from FROG measurements is shown by the dashed line. (b) Intensity and phase profiles at the center of the overlap region : numerical simulations (solid lines), fit by a black soliton (circles). (c) Ouput spectrum convoluted with the transfer function of the optical spectrum analyzer.

Fig. 4: Experimental setup used for the generation of the pair of pulses and for the self-similar Raman amplification.

Fig. 5: Intensity and chirp profiles retrieved from FROG measurements for the initial pulse (diamonds, $\mathrm{x}$ 10) and for the output pulse (gray circles). Experimental results are compared with 
linear and parabolic fits of the chirp and intensity profiles, respectively (dotted line) and with results from numerical simulations (solid line).

Fig. 6: (a) Autocorrelations of the pair of pulses at $z=5.3 \mathrm{~km}$ : experimental results (circles), numerical calculations (solid line), sinusoidal fit (dotted line). (b) Output experimental spectra for a single pulse (dashed gray line) and a pair of pulses (solid line).

Fig. 7: Evolutions of the repetition rate of the dark soliton train as a function of (a) the time separation between the initial pulses, (b) the initial pulse energy and (c) the integrated gain of the Raman amplifier. Experimental results (circles) are compared with numerical predictions of Eq. (6) (solid lines) and Eq. (1) (dotted lines) and with analytical predictions $f_{s}=C_{p} \Delta T$ (mixed lines).

Fig. 8: Propagation of three initial pulses in the Raman amplifier. (a) Intensity profiles at different distances of propagation. (b) Contour plot of the evolution of the normalized intensity profile of the dark soliton trains. (c) Intensity profiles of two colliding dark solitons at different propagation distances : before the collision (dashed line), during the collision (solid line) and after the collision (dotted line). (d) Contour plot of the evolution of the normalized intensity profile of two pairs of colliding dark solitons. The dashed gray line represents the evolution of one pair of dark solitons in the absence of collision. 
Fig. 1
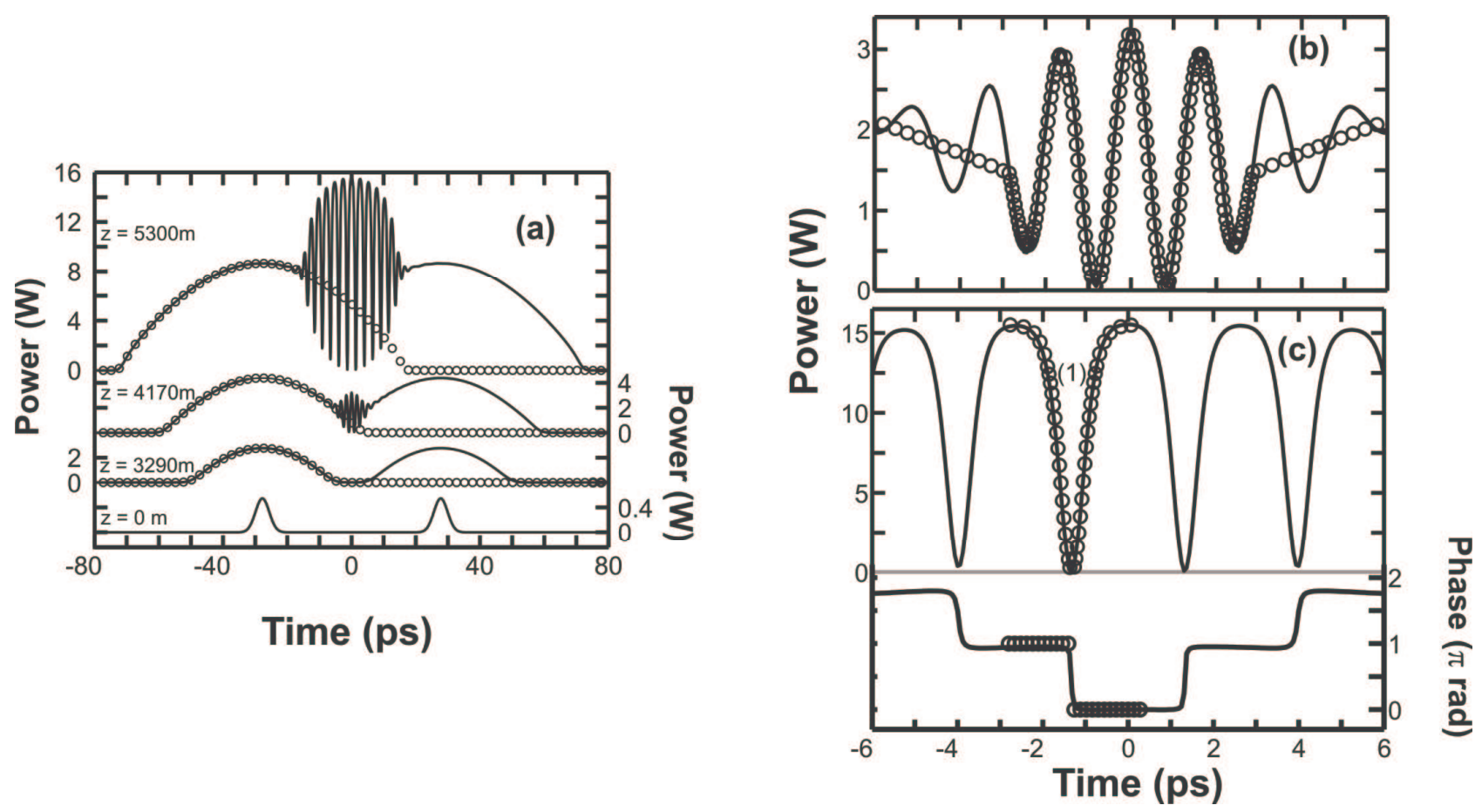
Fig. 2
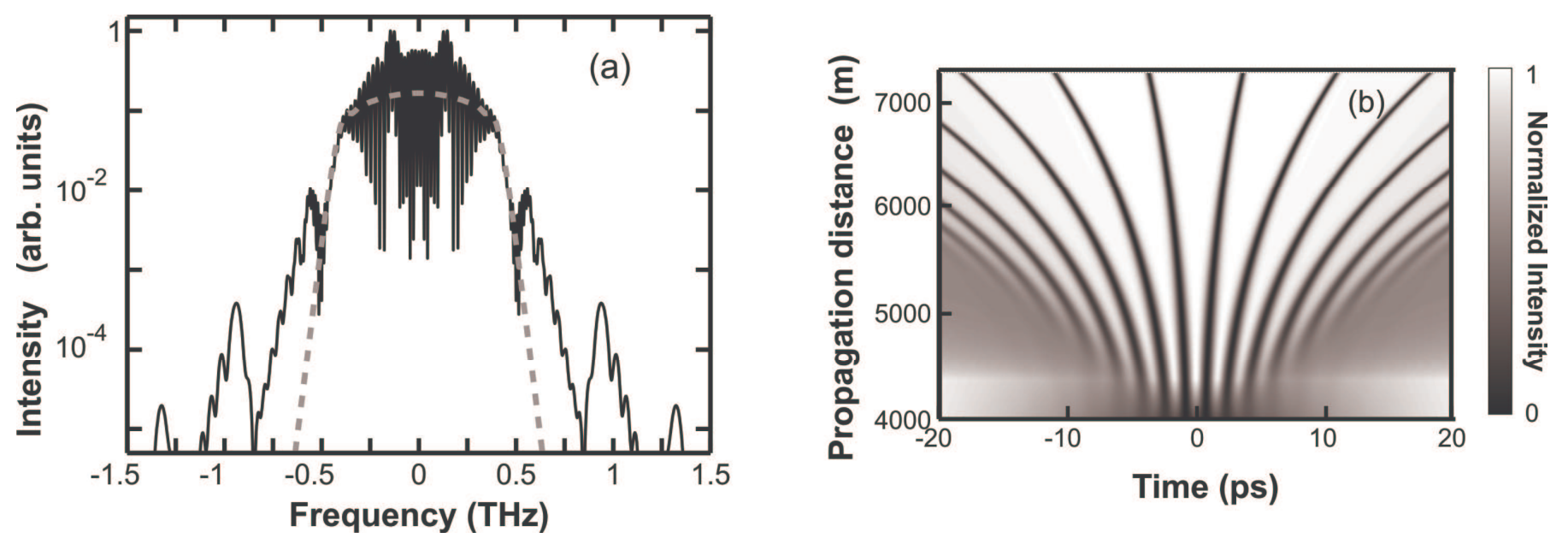
Fig. 3
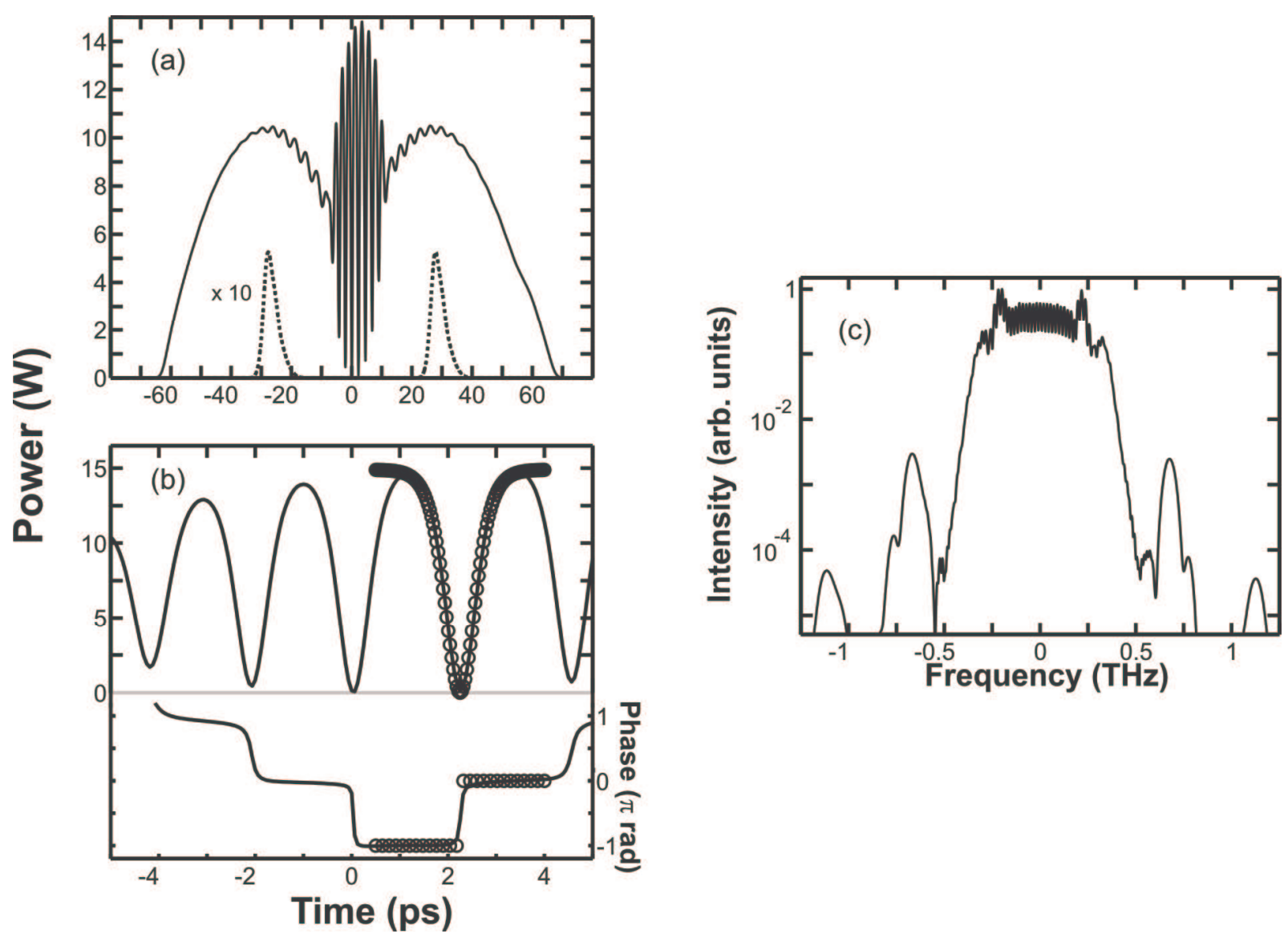


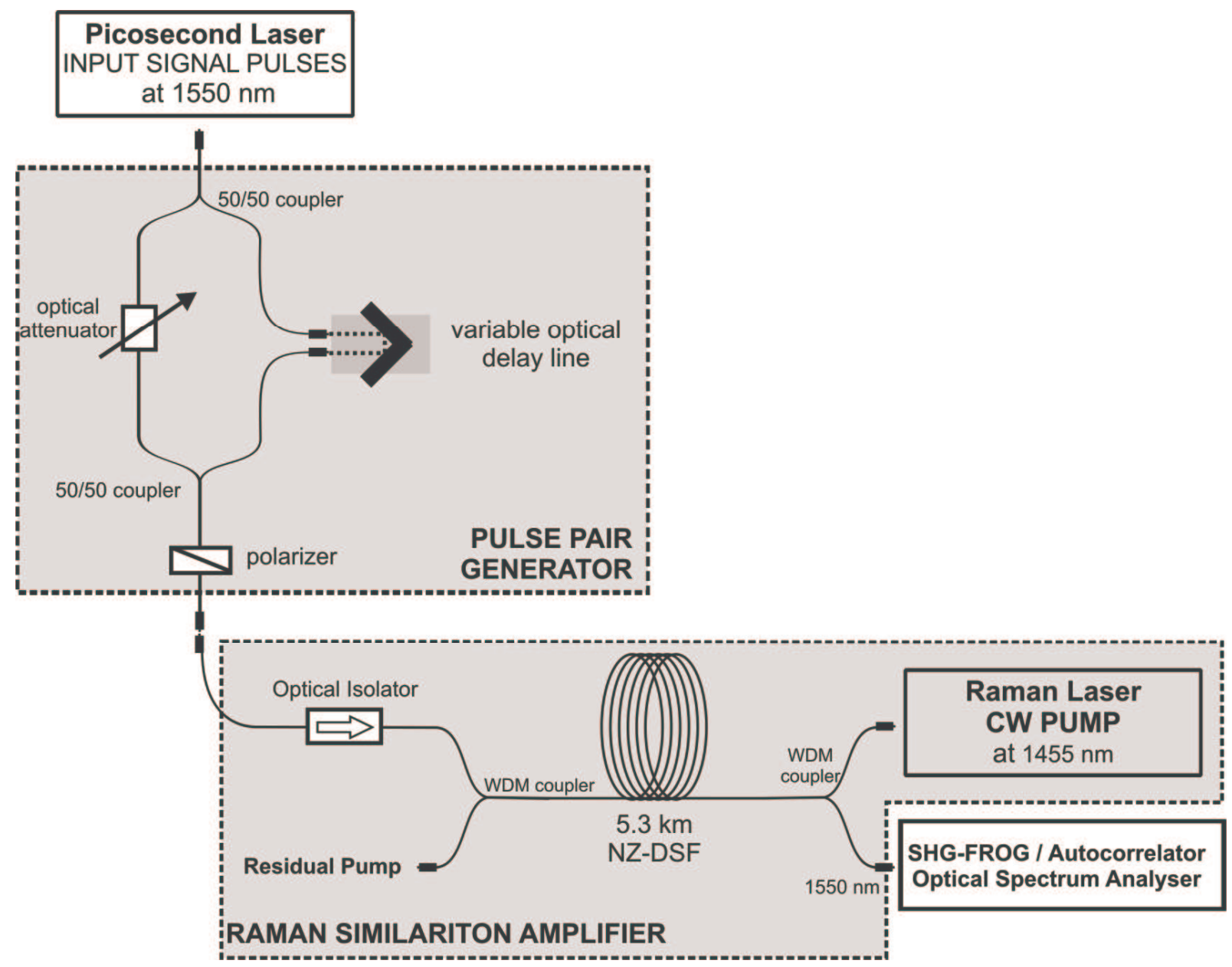


Fig. 5

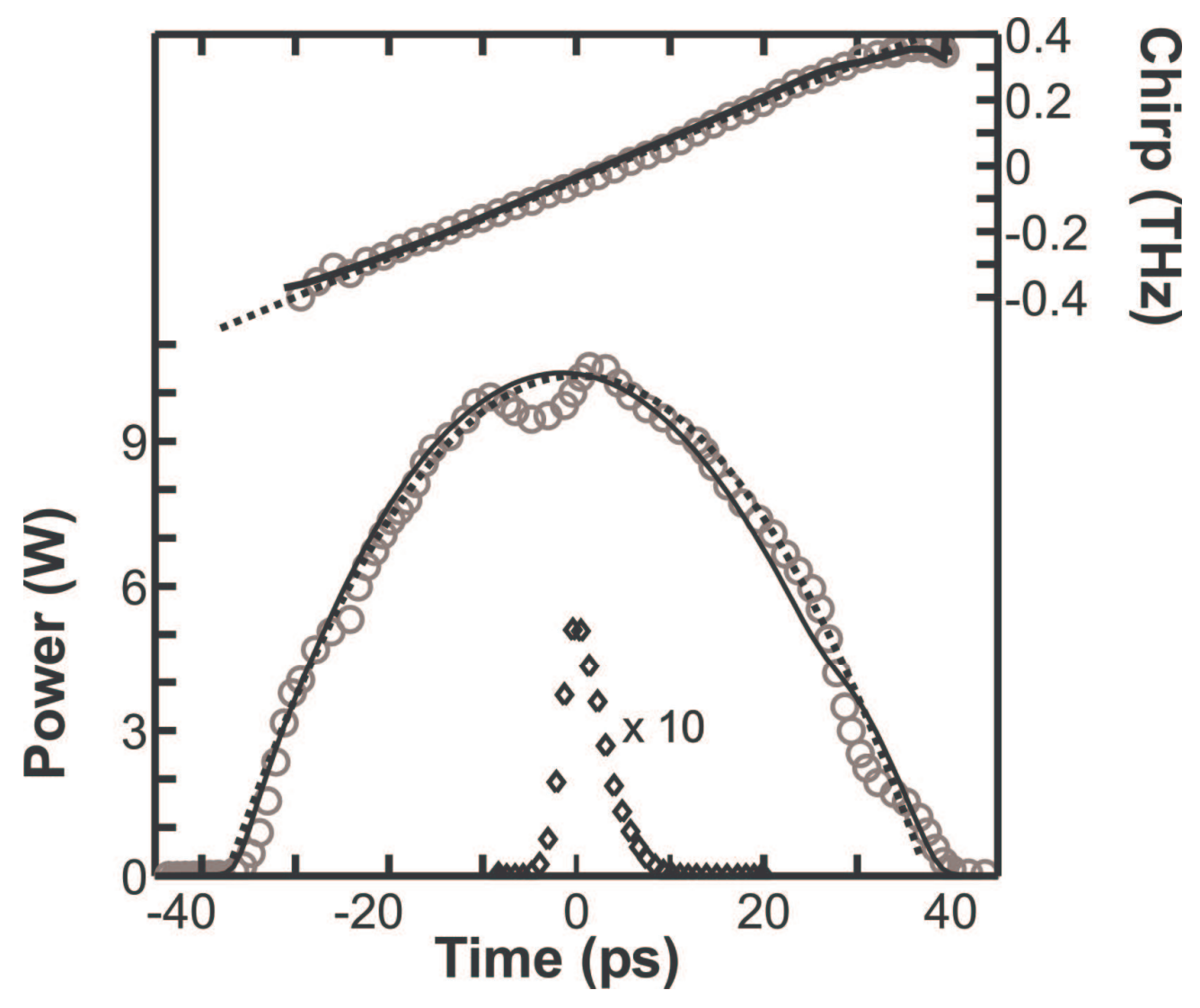


Fig. 6
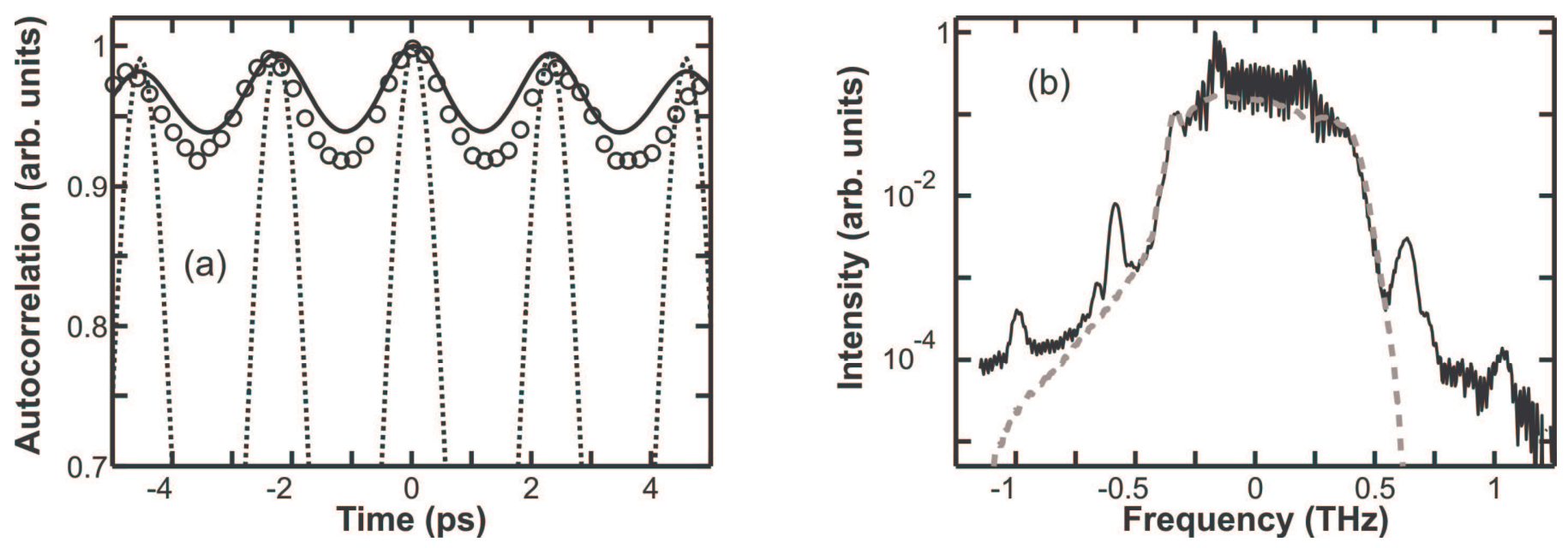
Fig. 7
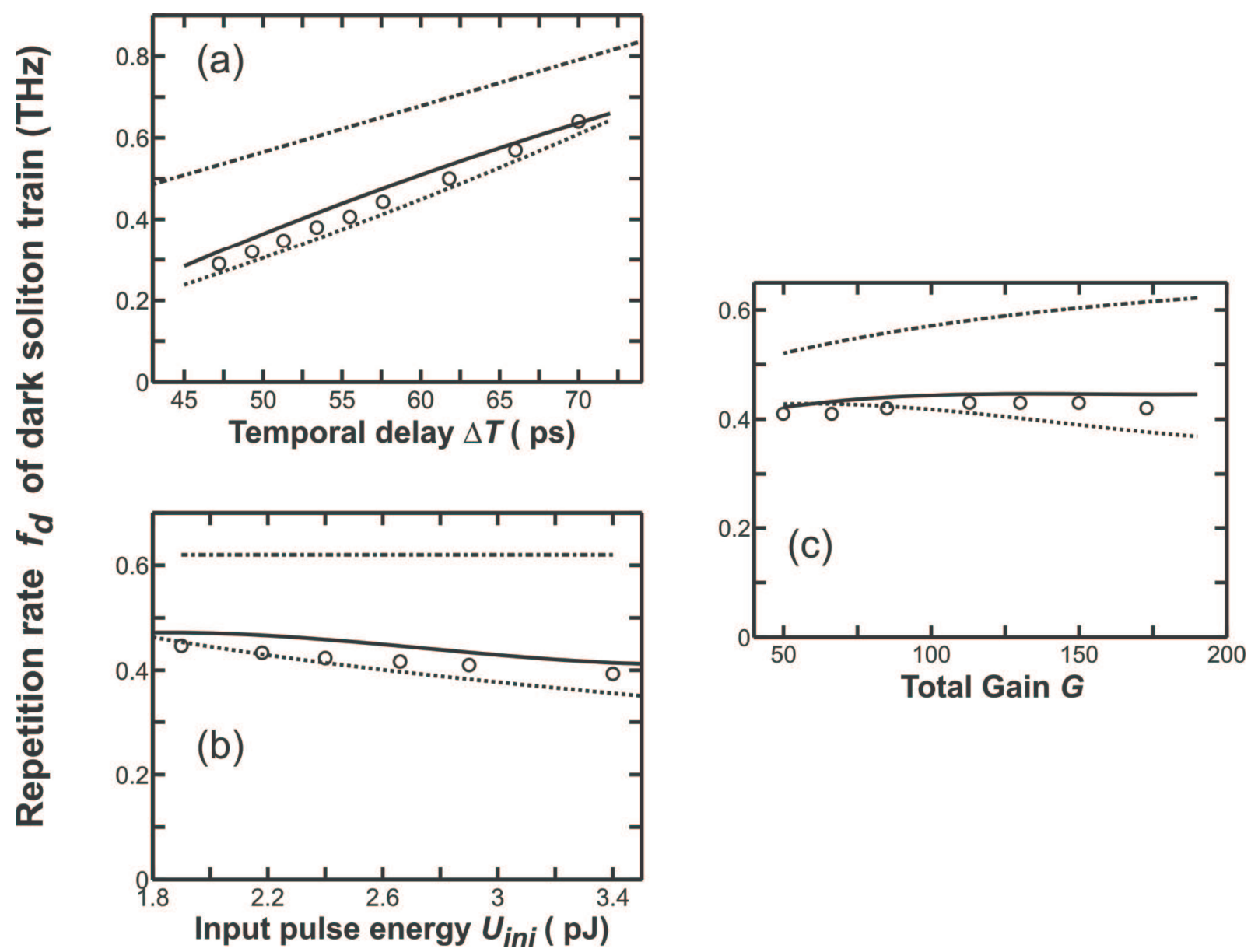

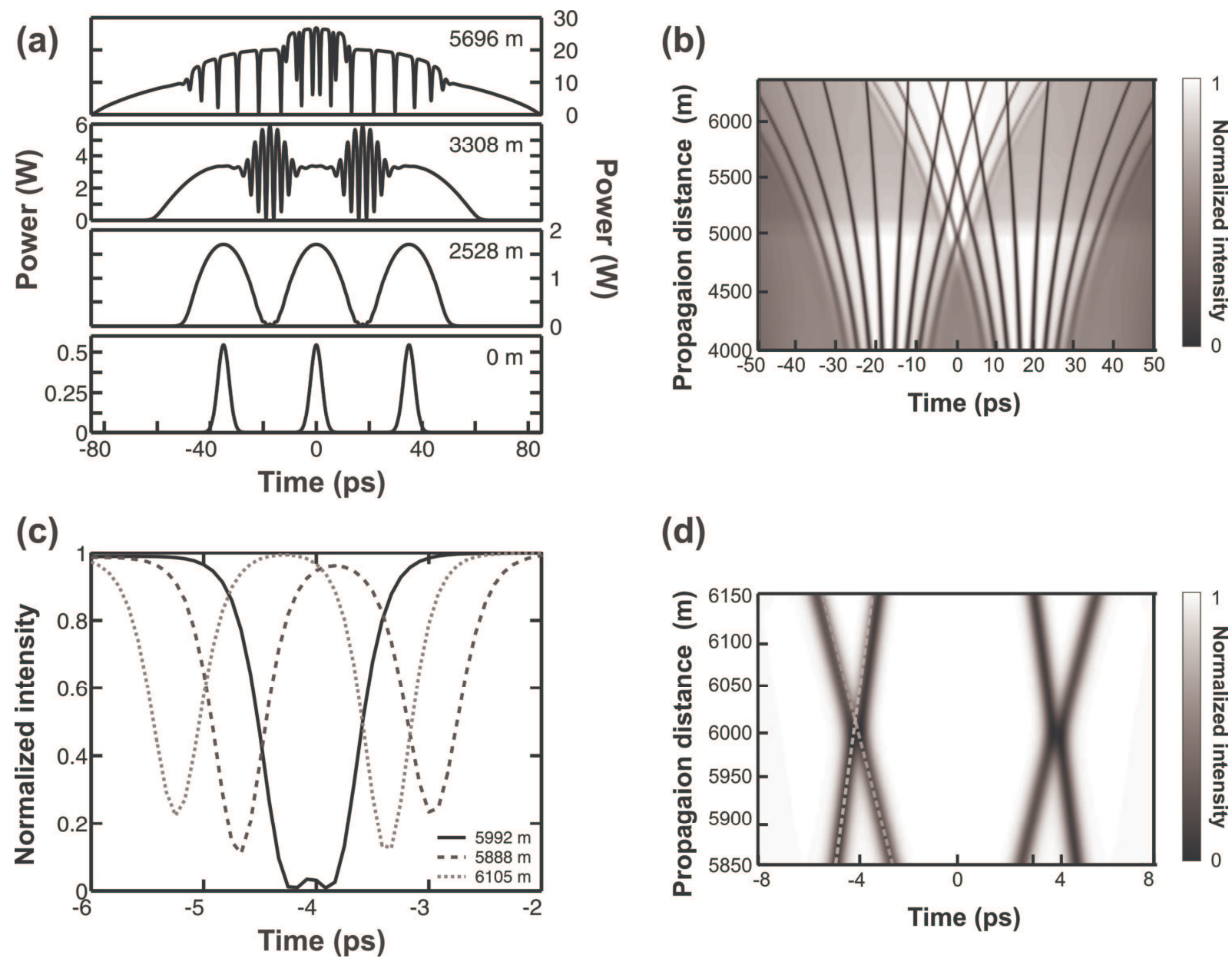

(d)

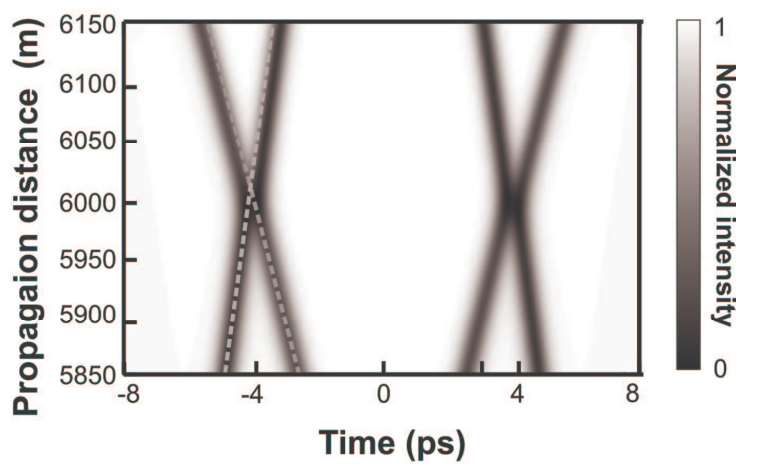

